\title{
Equilibrium Analysis in Imperfect Traders' and GenCos' Market
}

\author{
Puneet Chitkara, Member IEEE, and Jin Zhong, Member IEEE
}

\begin{abstract}
The paper models the strategic behavior of traders, GenCos and ISO using the multi-leader-follower framework. The outcomes of the strategic behavior of the players have been modeled using an equilibrium problem with equilibrium constraints. From a policy perspective it is seen that allowing the GenCos to hold FTRs may be welfare enhancing under certain demand conditions and ownership patterns of transmission rights and generation assets. The proposed model has been simulated on a 3 bus system.
\end{abstract}

Index Terms - Strategic behavior, multi-leader-follower games, Cournot conjectures, transmission rights, Equilibrium Problem with Equilibrium Constraints.

\section{INTRODUCTION}

$\mathrm{I}$ $\mathrm{N}$ the electricity markets where generators sell power to the grid at the market prices of their nodes, arbitragers and marketers can eliminate price differences between nodes [1]. However, Borenstein et al [2] show that traders' markets may not be competitive. The number of trading firms is limited because of the information asymmetry about the trading opportunities or certain legal and institutional barriers that govern the market. This provides market power to the traders and results in an imperfectly competitive market. Hence, in such markets, traders cannot be expected to trade to a point where the marginal trade breaks even. In a market with oligopolistic traders and generation companies (GenCos), each market participant makes strategic decision about its own price and output after considering the reactions of other players. The nature of such reaction depends on the conjectures that each player holds about the rival players.

A mathematical model is developed in this paper to formulate the equilibrium problem in the imperfect market with traders and GenCos. The model consists of two stages. We assume that, at each stage, all previous stage actions are observable to the players who base their current decisions on that information and on their rational expectation about the behaviors of all other players in the current stage and subsequent stage outcomes. The conjectural scheme regarding the GenCos' and traders' strategic behaviors is shown in Fig. 1.

This work was supported by the Research Grants Council of Hong Kong under Grant HKU 7196/07E.

P. Chitkara and J. Zhong are with the Department of Electrical and Electronic Engineering, the University of Hong Kong, Hong Kong (email: chitkara@eee.hku.hk, jzhong@eee.hku.hk)

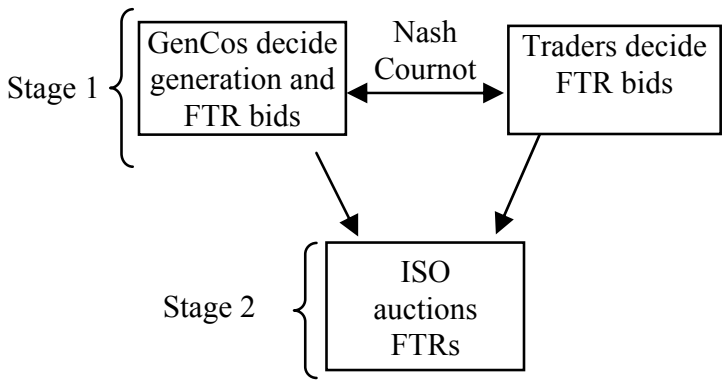

Fig. 1. GenCos and Traders anticipate the impacts of their decisions on net injections

The intent in this and related papers is not to predict whether or not market power would be exercised by any company. Rather, the analysis of the results is aimed at simulating conditions which might allow a GenCo to exercise market power in an imperfect market with oligopolistic traders and GenCos, as well as analyzing its impact on consumer surplus. Following [3]-[6], in this paper, we ignore price uncertainties and focus on the effects of the auction of transmission rights on equilibrium prices and quantities under different market structures, ownership patterns and conjectural assumptions, and hence on the market power of GenCos and consumer surplus.

In the literature on modeling strategic behavior in electricity networks, alternative representations have been used to model the conjectures of competing players. EPECs have been particularly used in game theoretic models of electricity markets over a network of generators and consumers [7]-[10]. EPECs arise when players face utility maximization problems in the form of MPECs [11]. MPECs have non-convex constraints and may have multiple local maxima. Thus EPECs may not have any (pure strategy) Nash equilibria in some instances [12]. Early algorithmic works on EPECs have focused on diagonalization techniques such as Gauss-Jacobi and Gauss-Seidel type methods. Such methods solve a cyclic sequence of MPECs, until the decision variable of all the MPECs reaches a fixed point. Sequential Nonlinear complementarity approach for solving EPEC has also been proposed [13]. In [14], Leyffer and Munson derive the nonlinear programming (NLP) formulation of the EPEC. The same has been used in [15] also. We use the approach suggested by Leyffer and Munson [14] to solve the EPEC problem for Nash-Cournot equilibrium in this paper.

The paper is organized as follows. The proposed model is formulated in Section II. In section III, the model is applied to 
a three-bus system. The simulation results are discussed. Section IV concludes.

\section{PROBLEM Formulation}

We illustrate the impacts of FTRs on market power of GenCos in the networks with loop power flows.

\section{A. The ISO's Problem}

In either of these games, players move sequentially. In stage 2, the ISO auctions transmission rights to those who value them the most. The value of the transmission right between a pair of nodes is defined in terms of the difference in nodal prices between the nodes. The price $p_{i}$ at node $i$ is given by the inverse demand function of the node, as shown in (1).

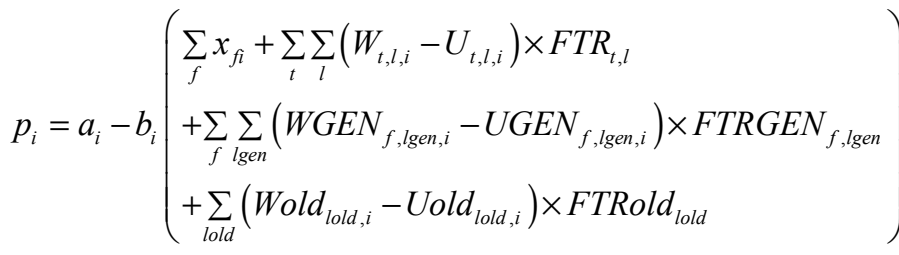

where,

$$
\begin{aligned}
& a_{i}: \text { the price intercept } \\
& \frac{a_{i}}{b_{i}}: \text { the intercept on the quantity axis }
\end{aligned}
$$

$x_{f i}$ is the generation output of the GenCo $f$ on node $i$

$F T R_{t, l}$ denotes FTR $l$ held by trader $t$

FTRGEN $_{f, \text { lgen }}$ denotes FTR lgen held by GenCo $f$

FTRold $_{\text {lold }}$ denotes the existing FTR lold

The directions of the new FTRs bid by the traders are denoted by $W$ and $U$.

$W_{t, l, i}=\left\{\begin{array}{lc}1 & \text { if node } \mathrm{i} \text { is the sink node for FTR } l \text { held by trader } t \\ 0 & \text { otherwise }\end{array}\right.$

$U_{t, l, i}=\left\{\begin{array}{cc}1 & \text { if node } i \text { is the source node for FTR } l \text { held by trader } t \\ 0 & \text { otherwise }\end{array}\right.$

The directions of the new FTRs bid by the GenCos are denoted by $W G E N$ and $U G E N$.

$W G E N_{f, \text { lgen }, i}= \begin{cases}1 & \begin{array}{l}\text { if node } i \text { is the sink node for FTR lgen held } \\ \text { by GenCo } f\end{array} \\ 0 & \text { otherwise }\end{cases}$

$U G E N_{f, \text { lgen }, i}= \begin{cases}1 & \begin{array}{l}\text { if node } i \text { is the source node for FTR lgen held } \\ \text { by GenCo } f\end{array} \\ 0 & \text { otherwise }\end{cases}$

Wold $_{\text {lold }, i}=\left\{\begin{array}{lc}1 & \text { if node } i \text { is the sink node for existing FTR lold } \\ 0 & \text { otherwise }\end{array}\right.$

Uold $_{\text {lold }, i}=\left\{\begin{array}{lc}1 & \text { if node } i \text { is the source node for existing FTR lold } \\ 0 & \text { otherwise }\end{array}\right.$

Power injections at all nodes are decided by the ISO in such a manner that the auctioned FTRs are simultaneously feasible. Following [16], we have defined net injections in terms of balanced point to point FTRs. Net injection at all nodes and therefore FTRs auctioned are the decision variables of the ISO. Given the nature of the inverse demand functions at all nodes, the directions of FTRs and volumes auctioned by the ISO can alter nodal energy prices. The value that a trader attaches to the FTR between a pair of nodes is defined in terms of the product of its bid parameter $\left(k_{t l}\right)$ and the difference of nodal prices [8]. Likewise, the GenCo may also wish to bid FTR, with a bid parameter kgen $_{f, \text { lgen }}$. Therefore, to auction FTRs to those, who value them the most, the ISO maximizes the following

$$
\begin{aligned}
& \underset{\text { FTR }_{t, l}, F T R G E N_{f, \text { gen }}}{\operatorname{Max}} Z_{\text {ISO }}=\sum_{t} \sum_{l} k_{t l}\left(F T R_{t, l} \times \sum_{i}\left(\left(W_{t, l, i}-U_{t, l, i}\right) \times p_{i}\right)\right) \\
& +\sum_{f} \sum_{\text {lgen }} \operatorname{kgen}_{f, \text { lgen }}\left(F T R G E N_{f, \text { lgen }} \times \sum_{i}\left(\left(W G E N_{f, \text { lgen }, i}-U G E N_{f, \text { lgen }, i}\right) \times p_{i}\right)\right)
\end{aligned}
$$

Subject to:

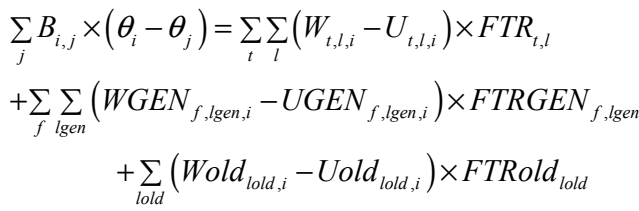

$B_{i, j} \times\left(\theta_{i}-\theta_{j}\right) \leq T_{i j}^{\max } \forall \operatorname{link} i j,\left(\mu_{i j}^{\max }\right)$

$-T_{i j}^{\max } \leq B_{i, j} \times\left(\theta_{i}-\theta_{j}\right) \forall$ link $i j,\left(\mu_{i j}^{\min }\right)$

FTR $_{t, l} \leq$ FTR $_{t, l}^{\max } \forall$ new FTR $l$ and trader $t\left(\alpha_{t l}^{\max }\right)$

$F_{T R}^{\min } \leq$ FTR $_{t, l} \forall$ new FTR $l$ and trader $t\left(\alpha_{t l}^{\min }\right)$

FTRGEN $_{f, \text { lgen }} \leq$ FTRGEN $_{f, \text { lgen }}^{\max }$

$\forall$ new FTR lgen and GenCo $f\left(\alpha g_{t l}^{\max }\right)$

$F_{T R G E N_{f, g e n}^{\min } \leq F T R G E N_{f, \text { gen }}}$

$\forall$ new FTR lgen and GenCo $f\left(\alpha g_{t l}^{\text {min }}\right)$

FTR $_{t, l}$, FTRGEN $_{f, \text { lgen }} \geq 0$

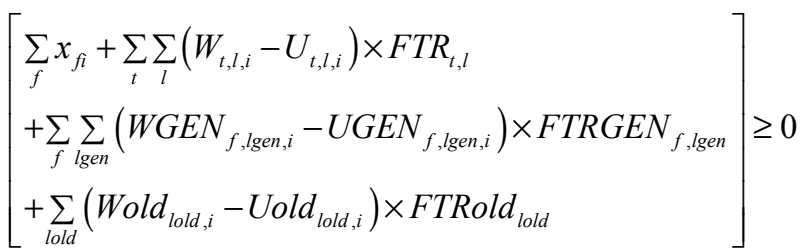

where, $p_{i}$ is the price defined in (1), $B_{i, j}$ is the susceptance of the line between nodes $i$ and $j, \theta_{i}$ is voltage angle at node $i$, $T^{m a x}$ is the maximum transmission capacity of transmission line between nodes $i$ and $j, F T R^{\max }{ }_{t l}$ and $F T R^{\min }{ }_{t l}$ are maximum and minimum values of FTR $l$ bid by trader $t$, and

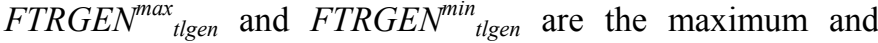
minimum values of FTR lgen bid by GenCo $f$.

The objective function (2) maximizes the product of the bidding parameters, FTR (MW) and the price difference between source and sink nodes for all new FTRs being sought by all the traders and all the GenCos. Equation (3) is the power balance constraint ${ }^{1}$ at each node $i$ using DC load flow

${ }^{1}$ Similar formulation albeit in a different context has been done by Gribik and Shirmohammadi [17] 
and defines power injections in terms of new FTRs auctioned to traders and GenCos and pre-committed old FTRs. Constraints (4) and (5) are transmission line capacity constraints. Inequalities (6) and (8), and (7) and (9) are constraints on the maximum and minimum values of the FTRs proposed to be purchased by traders and GenCos, respectively. All new FTRs auctioned are non-negative (10). The consumer at any node cannot self generate and hence, the demand at each node is constrained to be non-negative by (11). The dual multipliers of the constraints in the proposed optimization problem are represented by the symbols, $\lambda_{i}, \mu_{i j}{ }^{\max }$, $\mu_{i j}^{\min }, \alpha_{t l^{\max }}, \alpha_{t l}^{{ }^{\min }}, \alpha g_{f, \text { lgen }}{ }^{\max }, \alpha_{f, \text { lgen }}{ }^{\min }$ and $\gamma_{i}$. These are assumed to be the same for all the leaders as the ISO cannot price discriminate.

The ISO's optimization problem stated in (2)-(11) is parameterized in the level of generation $\left(x_{f i}\right)$ by each GenCo $f$ 's generator at node $i$, the bid parameters of GenCos $\left(\mathrm{kgen}_{f, \text { lgen }}\right)$, and the bid parameters of the traders $\left(k_{t l}\right)$.

\section{B. GenCo's problem}

The optimization problem for each GenCo $f$ is formulated as

$$
\begin{aligned}
& \underset{x_{f}, \text {,kgen }_{f, \text { Igen }}}{\operatorname{Max}_{f}} Z_{i \in G E N_{f}}\left[p_{i} \times x_{f i}-m c_{f i} \times x_{f i}\right] \\
& \left.+\sum_{\text {lgen }}\left(\left(1-\text { kgen }_{f, \text { lgen }}\right) \times F T R G E N_{f, \text { lgen }} \times \sum_{i}\left(W G E N_{f, \text { Igen }, i}-U G E N_{f, \text { lgen }, i}\right) \times p_{i}\right)\right)
\end{aligned}
$$

Subject to:

$$
\begin{aligned}
& x_{f i} \leq x_{f i}^{C A P} \quad \forall i, \quad\left(\rho_{f i}\right) \\
& \operatorname{kgen}_{f, \text { lgen }}^{\max }-\text { kgen }_{f, \text { lgen }} \geq 0 \quad \forall \text { lgen, } \quad\left(\delta_{f, \text { lgen }}^{\text {gmax }}\right) \\
& \text { kgen }_{f, \text { lgen }}-\operatorname{kgen}_{f, \text { lgen }}^{\text {min }} \geq 0 \quad \forall \text { lgen, } \quad\left(\delta_{f, \text { lgen }}^{\text {gmin }}\right) \\
& x_{f i} \geq 0
\end{aligned}
$$

where,

$p_{i}$ is the price defined as in (1)

$m c_{f i}$ is the marginal cost

$G E N_{f}$ denotes the set of all generators owned by GenCo $f$

$x_{f i}^{C A P}$ is the maximum generation capacity of generator $i$

$\rho_{f i}$ is the dual multiplier of the capacity constraint

Equations (14) and (15) are respectively the constraints on the maximum and minimum values of the strategic bid variable $\left(\mathrm{kgen}_{\text {flgen }}\right)$ of each FTR lgen sought by GenCo $f$. $\delta_{\text {flgen }}^{\max _{\text {Igen }}}$ and $\delta_{\text {flgen }}^{\text {max }}$ are the dual variables attached to these constraints, respectively.

\section{Trader's problem}

The FTRs held by traders are defined in terms of the power delivery at node $j$ and receipt at node $i$, as a specified amount of FTR (in MW) and a per MW price. Transmission rights considered here are akin to the prepaid forward contracts (or a call option with zero strike price), which are worth as much as or less than the underlying assets [18]. They are financial instruments issued by the ISO that entitle the transmission right holders to be reimbursed from the congestion charges collected by the ISO. The congestion charges are calculated in terms of the differences of prices at the sink and the source nodes. We ignore the risk due to the volatility in the difference in nodal prices. The same has been examined in [19]. Since the aim of the paper is to discuss the relationship between FTR auction and market power of GenCos, we concentrate on that issue alone following [3]-[6]. However, inclusion of risk and the impact of system contingencies in the model of traders and GenCos are intended to be taken up in the further research by the authors. Each FTR bidder assumes that the FTRs auctioned to it will be influenced by the FTRs auctioned to its rivals by the ISO in stage 2 under both the conjectural assumptions. The trader needs to decide bidding parameter, $k_{t, l}$ accordingly to maximize profits from holding FTRs. The trader does this by appending the KKT conditions of the ISO's problem (2)-(11) to its own problem given by (16) (18). Each trader will take the strategic bid parameters $\left(k_{t, l}\right)$ of its rivals as given (conjectures about rival FTR bidders' price bids have been considered in [19]). Also, it is assumed that different traders bid for FTRs between different/same pair of nodes.

Each trader chooses strategic bid parameter $k_{t, l}$ which maximizes the total profit (16) from all the FTRs proposed to be held by the trader between pre-decided pair of nodes (between the nodes of its interest). Hence the optimization problem for each trader $t$ to optimize all FTRs held by it is formulated as

$\left.\operatorname{Max}_{k_{t, l}} Z_{t}=\sum_{l}\left(1-k_{t, l}\right) \times F T R_{t, l} \sum_{i}(W(t, l, i)-U(t, l, i)) \times p_{i}\right)$

Subject to:

$k_{t, l}^{\max }-k_{t, l} \geq 0 \quad \forall l, \quad\left(\delta_{f, l}^{\max }\right)$

$k_{t, l}-k_{t, l}^{\text {min }} \geq 0 \quad \forall l, \quad\left(\delta_{t, l}^{\text {min }}\right)$

where, $p_{i}$ is the price defined as in (1).

Equations (17) and (18) are respectively the constraints on the maximum and minimum values of the strategic bid variable $\left(k_{t, l}\right)$ of each FTR $l$ sought by trader $t . \delta^{n a x}{ }_{t, l}$ and $\delta^{n i n}{ }_{t, l}$ are the dual variables attached to these constraints respectively.

The effect of traders submitting FTR bids as in above model is that the amount of power transmitted between nodes is determined by the aggregated bids finally auctioned by the ISO in stage 2. Since generators sell electricity at their nodes only, the power flow depends on the existing FTRs and the new FTRs auctioned. This is reflected in equation (3).

\section{Solution Method}

Scheme under conjecture assumption shown in Fig. 1, is a multi-leader, one follower game. The leaders are not only the GenCos but also the traders. All the GenCos and traders compete with each other in a Nash-Cournot manner.

Under this conjecture assumption, each leader, GenCo ((12)-(15)) and trader ((16)-(18)) takes the first order KarushKuhn-Tucker (KKT) conditions of the ISO's problem, (2)(11), as constraints in their optimization problems. Each leader therefore solves a Mathematical Problem with Equilibrium Constraints (MPEC). The first-order conditions for this generalized Nash Game are non-square in this case, because some multipliers cannot be matched to constraints. 
We assume that the multipliers on identical joint constraints are the same, that is, the shadow prices are set by the ISO that cannot price discriminate. Equilibrium between all the leaders can be obtained by solving all the MPECs for all the leaders simultaneously. This leads to the formulation of an Equilibrium Problem with Equilibrium Constraints (EPEC). Following Leyffer and Munson [14], we move all the complementarity conditions of this EPEC into the objective via the penalty approach and transform the problem into a nonlinear programming problem (NLP). Further, the Theorem 3.1 in [14] states that if a local solution is found to this NLP with the objective function value equal to zero, then the values of the decision variables of both the upper and lower level problems represent a strongly stationary point of the multileader-follower game. In all our simulations we have considered solutions with the objective function values less than $10^{-15}$. The simulations were done in GAMS [20] using CONOPT-3 solver.

\section{NumericAl EXAMPLES AND DiscusSIONS}

Three numerical examples are presented in this section to illustrate the applications of the proposed models. In each one of the numerical examples, the results and discussions are presented for three cases.

Case I: All traders and GenCos compete strategically. Each of them maximizes its own profit.

Case II: All traders collude and maximize their joint profit; all GenCos also collude and maximize their joint profit.

Case III: All GenCos and Traders collude and maximize the joint profit.

The examples have been analyzed to study the impacts of ownerships of FTRs by GenCos under alternative ownership patterns, demand conditions and conjecture assumptions on consumer surplus. This is important for design of regulatory policy, such as the one that restricts GenCos from possessing FTRs.

\section{A. Example A}

A three node network $(i=1,2,3)$ as shown in Fig. 2. Two generators owned by different GenCos $(f=1,2)$ are located at node 1 and node 2, respectively. In this example, assume that three traders $(t=1,2,3)$ and two GenCos participate in the FTR and power markets. Each trader bids for one FTR between different nodes. The FTRs $(l=1,2,3)$ are FTR 1 (from node 1 to 3 ), FTR 2 (from node 2 to 3 ) and FTR 3 (from node 2 to 1 ). Trader 1, Trader 2 and Trader 3 bid for FTR 1, FTR 2, and FTR 3, respectively, as shown in Fig. 2. The GenCos also bid for FTRs to serve the energy requirements at their nodes. GenCo 1 bids for FTR directed from node 3 to node 1 (FTRGEN 1) and GenCo 2 bids for FTR directed from node 1 to node 2 (FTRGEN 2).

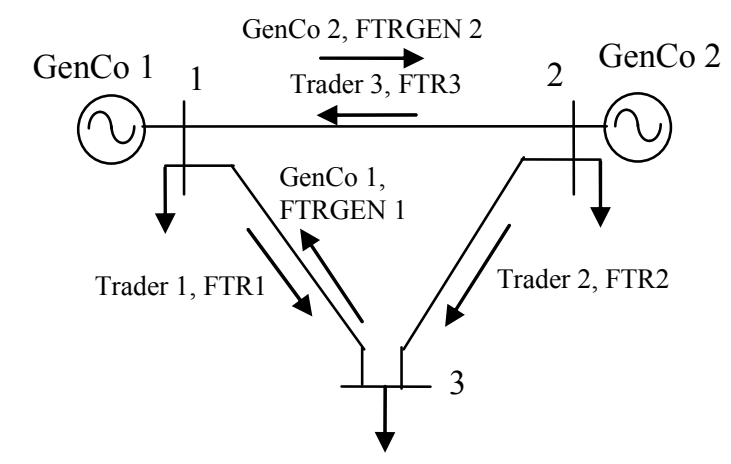

Fig. 2. Example A - three node system

The inverse demand functions of nodes 1, 2 and 3 are:

$$
\begin{aligned}
& \mathrm{p}_{1}=0.20-0.008 * \mathrm{q}_{1} \\
& \mathrm{p}_{2}=0.15-0.007 * \mathrm{q}_{2} \\
& \mathrm{p}_{3}=0.20-0.08 * \mathrm{q}_{3}
\end{aligned}
$$

where, $\left(\mathrm{p}_{1}, \mathrm{q}_{1}\right),\left(\mathrm{p}_{2}, \mathrm{q}_{2}\right),\left(\mathrm{p}_{3}, \mathrm{q}_{3}\right)$ are the price and demand pairs at the three nodes respectively. The generating unit at node 1 has a capacity of $550 \mathrm{MW}$, and a constant marginal cost $(\mathrm{mc})$ of $\$ 10 / \mathrm{MWh}$. The unit at node 2 has a capacity of $1500 \mathrm{MW}$ and a constant marginal cost of $\$ 8 / \mathrm{MWh}$.

The values of FTR ${ }^{\max }$ and FTRGEN ${ }^{\max }$ as in equations (6) and (8) respectively have been assumed uniformly as 2000 MW for all trades; and similarly $F T R^{\min }$ and $F T R G E N^{\text {min }}$ as in equations (7) and (9) respectively have been assumed to be zero. The FTR bidders expect to get the differences of the nodal prices as the maximum value per MW of FTR. The solutions to this example under the three cases are given in Table I and Table II.

\begin{tabular}{|c|c|c|c|c|}
\hline & & Case I & Case II & Case III \\
\hline \multirow{3}{*}{$\begin{array}{l}\text { Delivered } \\
\text { prices } \\
(\$ / \mathrm{MWh}) \\
\end{array}$} & $\mathrm{p}_{1}$ & 15.0 & 14.7 & 15.00 \\
\hline & $\mathrm{p}_{2}$ & 12.3 & 12.3 & 11.5 \\
\hline & $\mathrm{p}_{3}$ & 15.0 & 14.7 & 15.00 \\
\hline \multirow{3}{*}{$\begin{array}{c}\text { GenCo’s } \\
\text { profits }(\$ / h r)\end{array}$} & $f=1$ & 2362 & 2408 & 2344 \\
\hline & $f=2$ & 2584 & 2591 & 2516 \\
\hline & Total & 4946 & 4999 & 4860 \\
\hline \multirow{4}{*}{$\begin{array}{c}\text { Trader's } \\
\text { profits }(\$ / h r)\end{array}$} & $\mathrm{t}=1 / \mathrm{FTR} 1$ & 0 & 0 & 0 \\
\hline & $\mathrm{t}=2 / \mathrm{FTR} 2$ & 591 & 526.9 & 0 \\
\hline & $\mathrm{t}=3 / \mathrm{FTR} 3$ & 0 & 0 & 0 \\
\hline & Total & 591 & 526.9 & 0 \\
\hline \multirow{5}{*}{$\begin{array}{c}\text { Trading } \\
\text { profits of } \\
\text { GenCos }(\$ / \mathrm{hr})\end{array}$} & FTRGEN 1 & 0 & 0 & 0 \\
\hline & FTRGEN 2 & 0 & 0 & 0 \\
\hline & FTR $1^{* *}$ & \multirow{3}{*}{\multicolumn{2}{|c|}{$\begin{array}{l}\text { Applicable in Case III only } \\
\text { Applicable in Case III only } \\
\text { Applicable in Case III only }\end{array}$}} & 0 \\
\hline & FTR $2^{* *}$ & & & 141 \\
\hline & FTR $3^{* *}$ & & & 625 \\
\hline \multicolumn{2}{|c|}{ Consumer surplus $(\$ / \mathrm{hr})$} & 2258 & 2476 & 2594 \\
\hline
\end{tabular}

TABLE I EXAMPLE A: PRICES AND PROFITS

** All the FTRs with traders (in Case I and Case II) are considered to be with the cartel of GenCos and traders in Case III.

TABLE II

EXAMPle A: DEMAND, GeNERATION, TRADE VOLUMES AND POWER Flows

\begin{tabular}{|c|c|c|c|c|}
\hline \multicolumn{2}{|c|}{} & Case I & Case II & Case III \\
\hline Quantities & $\mathrm{q}_{1}$ & 625 & 664 & 625 \\
demanded & $\mathrm{q}_{2}$ & 392.42 & 392 & 500 \\
(MW) & $\mathrm{q}_{3}$ & 62.5 & 66 & 62.5 \\
\hline Generation by & $\mathrm{f}=1$ & 472.3 & 513.4 & 468.7 \\
GenCos (MW) & $\mathrm{f}=2$ & 607.6 & 608.4 & 718.7 \\
\hline \multirow{2}{*}{ FTR auctioned } & $\mathrm{t}=1$ /FTR 1 & 0 & 592.9 & 0 \\
(MW) & $\mathrm{t}=$ 2/FTR 2 & 215.2 & 216.8 & 0 \\
& $\mathrm{t}=$ 3/FTR 3 & 0 & 0 & 0 \\
\hline
\end{tabular}




\begin{tabular}{|c|c|c|c|c|}
\hline \multirow{2}{*}{$\begin{array}{c}\text { FTR } \\
\text { auctioned to }\end{array}$} & FTRGEN 1 & 152.7 & 743.3 & 653.9 \\
\cline { 2 - 5 } GenCos (MW) & FTRGEN 2 $^{*}$ & 0 & 0 & 0 \\
& FTR 2 2* $^{* *}$ & \multicolumn{2}{|c|}{ Applicable in Case III only } & 676.1 \\
& FTR 3 & \multicolumn{2}{|c|}{ Applicable in Case III only } & 40.2 \\
& Applicable in Case III only & 178.5 \\
\hline \multirow{2}{*}{ Power flows } & flow 1-2 & -116.6 & -116.8 & -118.7 \\
(MW) & flow 2-3 & 98.6 & $100^{*}$ & $100^{*}$ \\
& flow 1-3 & -36.1 & -33.6 & -37.5 \\
\hline
\end{tabular}

* Congested line.

** All the FTRs with traders (in Case I and Case II) are considered to be with the cartel of GenCos and traders in Case III.

\section{B. Example $B$}

In Example B the same three node network is considered. In addition, GenCo 1 owns not only the generator at node 1, but also a new generator at node 3; Trader 1 bids for an additional FTR, FTR 4 from node 2 to node 1, competing with Trader 2 that bids along the same corridor. The new generator and trade are shown with dotted lines in Fig. 3.

The demand function at each node remains the same as in Example A. The new generator at node 3 has a marginal cost of $\$ 9 / \mathrm{MWh}$. The maximum value of FTR 4 is $2000 \mathrm{MW}$. The results of the simulations are presented in Table III and Table IV.

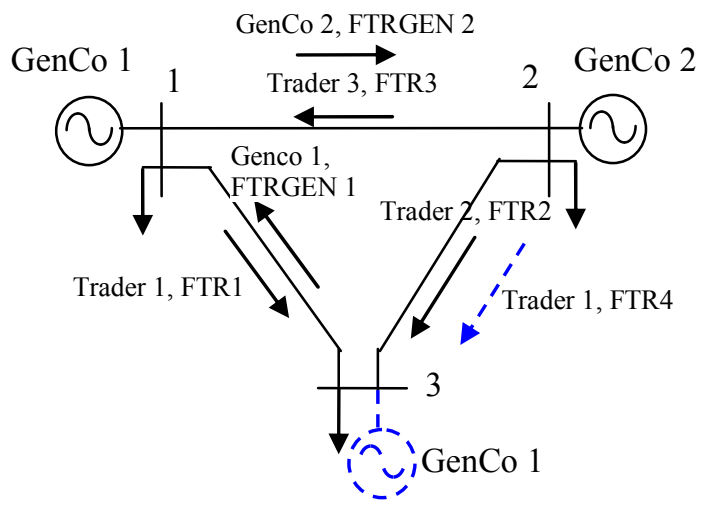

Fig. 3. Example B - three node system

TABLE III

EXAMPLE B: PRICES AND PROFITS

\begin{tabular}{|c|c|c|c|c|c|}
\hline & & & Case I & Case II & $\begin{array}{c}\text { Case } \\
\text { III }\end{array}$ \\
\hline \multirow{3}{*}{$\begin{array}{l}\text { Delivered } \\
\text { prices } \\
(\$ / \mathrm{MWh})\end{array}$} & \multirow{3}{*}{\multicolumn{2}{|c|}{$\begin{array}{l}\mathrm{p}_{1} \\
\mathrm{p}_{2} \\
\mathrm{p}_{3}\end{array}$}} & 14.17 & 14.17 & 15.00 \\
\hline & & & 12.23 & 12.23 & 11.5 \\
\hline & & & 14.45 & 14.5 & 14.5 \\
\hline \multirow{4}{*}{$\begin{array}{c}\text { GenCo’s } \\
\text { profits }(\$ / \mathrm{hr})\end{array}$} & \multirow{2}{*}{$\mathrm{f}=1$} & at 1 & 2173 & 2170 & 2069 \\
\hline & & at 3 & 372 & 378 & 402 \\
\hline & \multicolumn{2}{|c|}{$\mathrm{f}=2$} & 2557 & 2555 & 2474 \\
\hline & \multicolumn{2}{|c|}{ total } & 5102 & 5103 & 4945 \\
\hline \multirow{5}{*}{$\begin{array}{c}\text { Trader's } \\
\text { profits }(\$ / \mathrm{hr})\end{array}$} & \multirow{2}{*}{$\mathrm{t}=1$} & FTR 1 & 0 & 0 & 0 \\
\hline & & FTR 4 & 189.7 & 322 & 0 \\
\hline & \multicolumn{2}{|c|}{$\mathrm{t}=2 / \mathrm{FTR} 2$} & 2.670 & 0 & 0 \\
\hline & \multicolumn{2}{|c|}{$\mathrm{t}=3 /$ FTR 3} & 212.859 & 80.73 & 0 \\
\hline & \multicolumn{2}{|c|}{ total } & 405.137 & 403.65 & 0 \\
\hline \multirow{6}{*}{$\begin{array}{c}\text { Trading } \\
\text { profits of } \\
\text { GenCos }(\$ / h r)\end{array}$} & \multirow{2}{*}{\multicolumn{2}{|c|}{$\begin{array}{l}\text { FTRGEN } 1 \\
\text { FTRGEN } 2\end{array}$}} & 0 & 0 & 4 \\
\hline & & & 0 & 0 & 0 \\
\hline & \multicolumn{2}{|c|}{ FTR $1^{* *}$} & \multicolumn{2}{|c|}{ Applicable in Case III only } & 0 \\
\hline & \multicolumn{2}{|c|}{ FTR $2^{* *}$} & \multicolumn{2}{|c|}{ Applicable in Case III only } & 11 \\
\hline & \multicolumn{2}{|c|}{ FTR $3^{* *}$} & \multicolumn{2}{|c|}{ Applicable in Case III only } & 0 \\
\hline & \multicolumn{2}{|c|}{ FTR $4^{* *}$} & \multicolumn{2}{|c|}{ Applicable in Case III only } & 711 \\
\hline \multicolumn{3}{|c|}{ Consumer surplus (\$/hr) } & 2865 & 2864 & 2626 \\
\hline
\end{tabular}

TABLE IV

EXAmPle B: DEMAND, GENERATION, TRADE VOLUMES AND POWER Flows

\begin{tabular}{|c|c|c|c|c|c|}
\hline & & & Case I & Case II & $\begin{array}{c}\text { Case } \\
\text { III }\end{array}$ \\
\hline \multirow{3}{*}{$\begin{array}{c}\text { Quantities } \\
\text { demanded } \\
\text { (MW) }\end{array}$} & \multirow{3}{*}{\multicolumn{2}{|c|}{$\begin{array}{l}\mathrm{q}_{1} \\
\mathrm{q}_{2} \\
\mathrm{q}_{3}\end{array}$}} & 728.8 & 729.2 & 625 \\
\hline & & & 395.6 & 395.8 & 500 \\
\hline & & & 69.4 & 68.7 & 68.75 \\
\hline \multirow{3}{*}{$\begin{array}{l}\text { Generation by } \\
\text { GenCos (MW) }\end{array}$} & \multirow{2}{*}{$\mathrm{f}=1$} & at 1 & 550 & 520.8 & 413.7 \\
\hline & & at 3 & 68.4 & 68.7 & 73.1 \\
\hline & \multicolumn{2}{|r|}{$\mathrm{f}=2$} & 551.3 & 604.2 & 706.9 \\
\hline \multirow{4}{*}{$\begin{array}{c}\text { FTR } \\
\text { auctioned } \\
\text { (MW) }\end{array}$} & \multirow{2}{*}{$\mathrm{t}=1$} & FTR 1 & 0 & 0 & 0 \\
\hline & & FTR 4 & 97.8 & 166.7 & 0 \\
\hline & \multicolumn{2}{|c|}{$\mathrm{t}=2 /$ FTR 2} & 1.2 & 0 & 0 \\
\hline & \multicolumn{2}{|c|}{$\mathrm{t}=3 /$ FTR 3} & 109.8 & 41.7 & 0 \\
\hline \multirow{6}{*}{$\begin{array}{c}\text { FTR auctioned } \\
\text { to GenCos } \\
(\mathrm{MW})\end{array}$} & \multirow{2}{*}{\multicolumn{2}{|c|}{$\begin{array}{l}\text { FTRGEN } 1 \\
\text { FTRGEN } 2\end{array}$}} & 0 & 0 & 8 \\
\hline & & & 0 & 0 & 0 \\
\hline & \multicolumn{2}{|c|}{ FTR $1^{* *}$} & \multicolumn{2}{|c|}{ Applicable in Case III only } & 0 \\
\hline & \multicolumn{2}{|c|}{ FTR $2^{* *}$} & \multicolumn{2}{|c|}{ Applicable in Case III only } & 3.7 \\
\hline & \multicolumn{2}{|c|}{ FTR $3^{* *}$} & \multicolumn{2}{|c|}{ Applicable in Case III only } & 0 \\
\hline & \multicolumn{2}{|c|}{ FTR $4^{* *}$} & \multicolumn{2}{|c|}{ Applicable in Case III only } & 203 \\
\hline \multirow{3}{*}{$\begin{array}{l}\text { Power flows } \\
\text { (MW) }\end{array}$} & \multirow{3}{*}{\multicolumn{2}{|c|}{$\begin{array}{l}\text { Flow 1-2 } \\
\text { Flow 2-3 } \\
\text { Flow 1-3 }\end{array}$}} & $-125^{*}$ & $-125^{*}$ & $-125^{*}$ \\
\hline & & & 83.7 & 83.3 & 81.9 \\
\hline & & & -82.5 & -83.3 & -86.3 \\
\hline
\end{tabular}

* Congested line.

** All the FTRs with traders (in Case I and Case II) are considered to be with the cartel of GenCos and traders in Case III.

In Case I of Example A (Table I), it is important to note that the prices at nodes differ, although there is no congestion in this case (see Table II, last row - power flows). Cournot prices differ at various nodes, as pointed out by Hobbs in [1], because of the imperfect arbitrage between nodes.

\section{Discussions}

The results of three cases listed in Tables I-IV are analyzed in the following.

1) Impact of FTR auctions to GenCos on consumer surplus

In Example B, consumer surplus is the highest in Case I (Table III). However, in Example A, it is the highest in Case III (Table I). It is also noted that possession of FTRs by GenCos (denoted by FTRGEN) in Case I and Case II in both the examples does not generate any profits for them (se e Table I for Example A, Table III for Example B)). However, in Case III in both the examples, the cartel formed by the collusion of GenCos and traders is able to alter the generation profile in a manner such that its overall profit from generation assets and FTRs is maximized. A comparison of Case III with the other two cases in Table II and Table IV show that the generation is increased at node 2 (cheap node, $m c=$ $\$ 8 / \mathrm{MWh})$ ) and reduced at node 1 (costly node, $m c=$ $\$ 10 / \mathrm{MWh}$ ). This is not only because the generation at the node 2 is cheaper than node 1 , but also because increased generation at node 2 helps in reducing the price at that node (refer Table I, $\mathrm{p}_{2}=\$ 11.5 / \mathrm{MWh}$ in Case III, as compared to $\$ 12.3$ in other two cases and similarly in Table III) and reduced generation at node 1 helps in increasing the price at node 1. This helps the cartel of GenCos and traders in Case III to maximize the profit from possession of FTRs. Incidentally, while in Example A, this helps in increasing consumer surplus of Case III (Table I, last row), in Example B, this leads to the least consumer surplus in Case III as compared to other cases 
(Table III, last row). Hence, from a policy point of view, it may not be prudent to impose a regulation that prevents GenCos from possessing transmission rights under all conditions of system operation.

2) Impact of spatial diversity of demand elasticity on aggregate consumer surplus

Further, economic regulations are meant to improve performance in terms of consumer surplus. "Performance", when measured in terms of consumer surplus, need not connote a higher aggregate demand served. This happens because of the diversity in the elasticity of demand across nodes - the spatial diversity of demand elasticity. As an illustration: the total demand served, in Example B is almost equal in Case I $(1193.8 \mathrm{MW}=728.8+395.6+69.4$, column 3 , row 2, Table IV), Case II $(1193.7 \mathrm{MW}=729.2+395.8+68.7$, column 4, row 2, Table IV) and in Case III (1193.75 MW = $625+500+68.75$, column 5, row 2, Table IV). However, the consumer surpluses in Case I and Case II are greater than that in Case III (see Table III, last row). Therefore the performance measured in terms of aggregate consumer surplus needs to take into account the spatial inequities which are innate in such measures. This could probably be done by assigning weights to the consumer surplus at various nodes - which opens up other issues pertaining to development of "optimal" weights.

\section{3) Inducement for cartel formation}

In both the examples discussed above, it is observed that the ownerships of FTRs generate the highest profit in Case III. For example in Table I, the sum of GenCo's total profit, trader's total profit and trading profit of GenCos equal to $(4946+591=5537)$ for Case I, $(4999+526=5525.9)$ for Case II, and $(4860+141+625=5626)$ for Case III, and similarly in Table III. However, all the traders in FTRs and GenCos do not gain uniformly from collusion. Hence the cartel may not materialize unless some bargaining mechanism ensures sharing of benefits between various players based on their contribution to the collusion. In other words, if some players (traders or GenCos) cause externalities for other players, the interested parties can bargain to correct any externality. In the small three network example it is probably possible to identify and negotiate. In a real power system such a negotiation may be costly or even impractical. In Example A, consider Table I, the gains and losses of each play by changing from Case II (cartel of traders vs. cartel of GenCos) to Case III (cartel of all traders and GenCos) are calculated as following.

- Trader 3 gains $\$ 625 / \mathrm{hr}$ from FTRs: [column 5, row 16 (625)] - [column 4, row $10(0)$ ];

- GenCo 1 loses \$64/hr: [column 4, row 5 (2408)] [column 5, row 5 (2344)];

- GenCo 2 loses \$75/hr: [column 4, row 6 (2591)] [column 5, row 6 (2516)];

- Trader 2 loses \$386/hr: [column 4, row 9 (526.9)] [column 5, row 15 (141)];

The total loss of GenCo 1 , GenCo 2 and Trader 2 is $\$ 525 / \mathrm{hr}$. Trader 3 could therefore redistribute its gain among the cartel partners and make every player better-off.
Assuming no transaction cost, this gain could be redistributed between the players so that no one loses from the collusion. As illustrated in Section III-C.1), such collusion is consumer welfare improving in Example A and consumer welfare reducing in Example B. Hence under this condition the regulator needs a market oversight to contain such transactions only if they result in the reduction of consumer surplus.

This discussion underscores that regulations restricting the GenCos from purchasing FTRs may not induce efficiency under all conditions of the ownership patterns and demand conditions. Further, the regulator may not be able to observe tacit collusion between players, thereby calling the effectiveness of such regulations into question.

\section{CONCLUSIONS}

The paper models the interaction between an imperfectly competitive traders' market and an imperfectly competitive GenCos' market conjecture assumption. The results of the models have implications for policy planners and regulators on the choices of market structures and design of regulatory mechanisms. The implications are that the possession of FTRs by GenCos may be welfare enhancing under certain conditions and hence regulations prohibiting GenCos from possessing FTRs could in fact reduce welfare.

\section{REFERENCES}

[1] B.F. Hobbs, "Linear Complementarity Models of Nash-Cournot Competition in Bilateral and POOLCO Power Markets," IEEE Trans. on Power Systems, Vol. 16, No. 2, pp. 194-202, 2001.

[2] S. Borenstein, J. Bushnell, C.R. Knittel, and C. Wolfram, "Inefficiencies and Market Power in Financial Arbitrage: A Study of California's Electricity Markets," University of California Energy Institute working paper CSEMWP-138, 2004.

[3] J. Bushnell, "Transmission Rights and Market Power," Electricity Journal, October, pp.77-85, 1999.

[4] S.S. Oren, "Economic Ineffciency and Passive Transmission Rights in Congested Electricity Systems with Competitive Generation," Energy Journal, Vol. 18, No. 1, pp. 63-83, 1997.

[5] W. Hogan, "Contract Networks for Electric Power Transmission," Journal of Regulatory Economics, Vol.4, No. 3, pp. 211-242, 1992.

[6] P. Joskow and J. Tirole, "Transmission Rights and Market Power in electric power networks," RAND Journal of Economics, Vol. 31, No. 3, pp. $450-487,2000$.

[7] C. A. Berry, B. F. Hobbs, W. A. Meroney, R. P. O'Neill, and W. R.Stewart Jr, "Understanding how market power can arise in network competition: a game theoretic approach," Utilities Policy, vol. 8, pp.139-158, 1999.

[8] J. Cardell, C. Hitt and W.W. Hogan, "Market Power and Strategic Interaction in Electricity Networks", Resource and Energy Economics, Vol. 19, pp. 109-137, 1997

[9] J. Yao, I. Adler, and S. Oren, "Modeling and computing two-settlement oligopolistic equilibrium in a congested electricity network," Department of IEOR, University of California, Berkeley, California," Manuscript, 2005

[10] X. Hu, D. Ralph, E. Ralph, P. Bardsley, and M. Ferris, "Electricity generation with looped transmission networks: bidding to an iso," University of Cambridge, The CMI Electricity Project, Department of Applied Economics, Cambridge, UK, working paper CMI EP 65, revised 2004.

[11] D. Ralph, and Y. Smeers, "EPECs as models for electricity markets", Power Systems Conference and Exposition, 2006. 
[12] X. Hu and D. Ralph, "Using EPECs to model bilevel games in restructured electricity markets with locational prices," University of Cambridge, Judge Business School, Cambridge, UK, Manuscript, 2005.

[13] Che-Lin Su, "A Sequential NCP Alghorithm for solving equilibrium problems with equilibrium constraints", Technical Report, Department of Management Science and Engineering, Stanford University, 2004.

[14] S. Leyffer and T. Munson, "Solving multi-leader-follower games," Preprint Argonne National Laboratory, vol. ANL/MCS-P1243-0405, Apr. 2005.

[15] G. Bautista, M.F. Anjos, and A. Vannelli, "Formulation of Oligopolistic Competition in AC Power Networks: An NLP Approach," IEEE Trans. on Power Systems, Vol. 22, No.1, pp. 105-115, 2007

[16] W. Hogan, J. Rosellon, and I. Vogelsang, "Toward a Combined Merchant-Regulatory Mechanism for Electricity Transmission Expansion," Conference Proceedings, 9 ${ }^{\text {th }}$ IAEE European Energy Conference, Florence, Italy, 2007.

[17] P.R. Gribik, D. Shirmohammadi, J.S. Graves, and J.G. Kritikson, "Transmission Rights and Transmission Expansions," IEEE Trans. On Power Systems, Vol. 20, No. 4, pp. 1728-1737, 2005.

[18] M. Liu and G. Gross, "Framework for the design and analysis of congestion revenue rights," IEEE Trans. on Power Systems, Vol. 19 No. 1, pp. 243-251, 2004.

[19] T. Li and M. Shahidehpour, "Risk Constrained FTR Bidding Strategy in Transmission Markets," IEEE Trans. on Power Systems, Vol. 20, No.2, pp. 1014-1021, 2005
[20] GAMS Release 2.50, “A user's guide," GAMS Development Corporation, 2006.

\section{BIOGRAPHIES}

Puneet Chitkara (M'07) received his B.E. degree in Electrical Engineering from Maulana Azad National Institute of Technology, Bhopal, India, in 1993, and the Ph.D degree in Economics from Indira Gandhi Institute of Development Research, Mumbai, India, in 1999. At present, he is a Senior Research Associate in the Department of Electrical and Electronic Engineering of the University of Hong Kong. His areas of interest are regulatory economics, applications of game theory to power markets and electricity sector deregulation.

Jin Zhong ( $\mathrm{S}^{\prime} 00$, M'04) received her B.Sc. degree from Tsinghua University, Beijing, China, in 1995, the M. Sc. degree from China Electric Power Research Institute in 1998 and the Ph.D degree from Chalmers University of Technology, Gothenburg, Sweden, in 2003. At present, she is an Assistant Professor in the Department of Electrical and Electronic Engineering of the University of Hong Kong. Her areas of interest are electricity sector deregulation, ancillary service pricing and power system planning. 